\title{
Designing Thermal Feedback for Notifying Users About Stress
}

\author{
Romina Poguntke \\ Romina.Poguntke@vis.uni-stuttgart.de \\ University of Stuttgart \\ Stuttgart, Germany \\ Albrecht Schmidt \\ Albrecht.Schmidt@ifi.lmu.de \\ Ludwig-Maximilians-University Munich \\ Munich, Germany
}

\author{
Jonathan Ilk \\ Ilk.Jonathan@gmail.com \\ University of Stuttgart \\ Stuttgart, Germany \\ Yomna Abdelrahman \\ yomna.eldin@gmail.com \\ Bundeswehr University Munich \\ Munich, Germany
}

\begin{abstract}
Though many new technologies have been adopted for stress detection, communicating stress to the users is still experienced primarily through visual or auditory channels. However, these commonly used feedback channels are already associated with smartphone notifications. Instead, we focus on thermal feedback yielding the advantage to preserve privacy due to its unobtrusiveness. By this work, we contribute an investigation of thermal feedback for notifying users about stress comprising the exploration of the preferred temperature level, rate of change, and body location. Accordingly, we compared different stimuli for each for those in a user study involving 21 participants. From their quantitative and qualitative feedback, we found that cold stimuli are preferred in general, showing that $\pm-0.5^{\circ} \mathrm{C}$ is the optimal rate of change and preferably when presented at the lower back. We conclude with discussing our findings from both the quantitative and qualitative data and finally present our research agenda paving the way for thermal feedback as a stress notifier.
\end{abstract}

\section{CCS CONCEPTS}

- Human-centered computing $\rightarrow H C I$ design and evaluation methods.

\section{KEYWORDS}

Thermal feedback, stress, temperature, body location

\section{ACM Reference Format:}

Romina Poguntke, Jonathan Ilk, Albrecht Schmidt, and Yomna Abdelrahman. 2019. Designing Thermal Feedback for Notifying Users About Stress. In PervasiveHealth '19: 13th EAI International Conference on Pervasive Computing Technologies for Healthcare, fune 20-23, 2019, Trento, Italy. ACM, New York, NY, USA, 5 pages. https://doi.org/10.1145/1122445.1122456

Permission to make digital or hard copies of all or part of this work for personal or classroom use is granted without fee provided that copies are not made or distributed for profit or commercial advantage and that copies bear this notice and the full citation on the first page. Copyrights for components of this work owned by others than the author(s) must be honored. Abstracting with credit is permitted. To copy otherwise, or republish, to post on servers or to redistribute to lists, requires prior specific permission and/or a fee. Request permissions from permissions@acm.org.

Pervasive Health '19, June 20-23, 2019, Trento, Italy

(c) 2019 Copyright held by the owner/author(s). Publication rights licensed to ACM ACM ISBN 978-1-4503-9999-9/18/06 . .\$15.00

https://doi.org/10.1145/1122445.1122456

\section{INTRODUCTION AND BACKGROUND}

Stress has been widely explored in many disciplines and for many purposes. While often the negative consequences are in the focus of media and societal discussions, research has been occupied with sensing stress $[1,5,7,12,13]$. Apart from medical stress detection approaches and work in human-computer interaction (HCI) deducing stress from, e.g. heart rate activity [2, 5, 9], prior work has researched biofeedback largely. Applications representing physiological signals have been used previously to notify users about their stress level [3]. However, often such applications require the user's visual attention and are prone to privacy violating incidences due to its visibility for bystanders. Further, tactile feedback, such as vibration or compression is already associated with smartphone or wrist-worn wearable notifications [8]. To provide a privacy securing but noticable and unobtrusive feedback, we investigate thermal feedback for notifying users about stress. Building upon previous work [14], we conducted a user study involving 21 participants to evaluate (a) the most suitable temperature level, (b) the best rate of change, and (c) the preferred body location for presenting thermal stress feedback.

\section{DESIGNING OPTIMAL THERMAL STRESS FEEDBACK}

Due to its unique advantages, such as unobtrusiveness, non-binary character and privacy preservation, we aimed to investigate the potential of thermal feedback for notifying users about their stress level. Accordingly, we differentiated between three factors determining the suitability of such a stimulus: temperature level, rate of change, body location. When designing the temperature values, we followed the recommendation of Wilson [14] showing that $\pm 1^{\circ} \mathrm{C}$ degrees, $\pm 3^{\circ} \mathrm{C}$ degrees, and $\pm 6^{\circ} \mathrm{C}$ degrees are perceived best for conveying emotional value. Simultaneously, we were inspired by [14] when choosing the rates of change. Further, we considered the optimal body location for presenting thermal. For this, we evaluated five different spots covering the upper body part, i.e. upper chest and lower back and the limbs, namely the foot arch as well as the upper and lower arm respectively the wrist $[10,11]$.

\section{EVALUATION}

For exploring the preferred thermal feedback for tactile stress notifications, we compared five temperature levels, three rates of change, and five distinct body locations. 


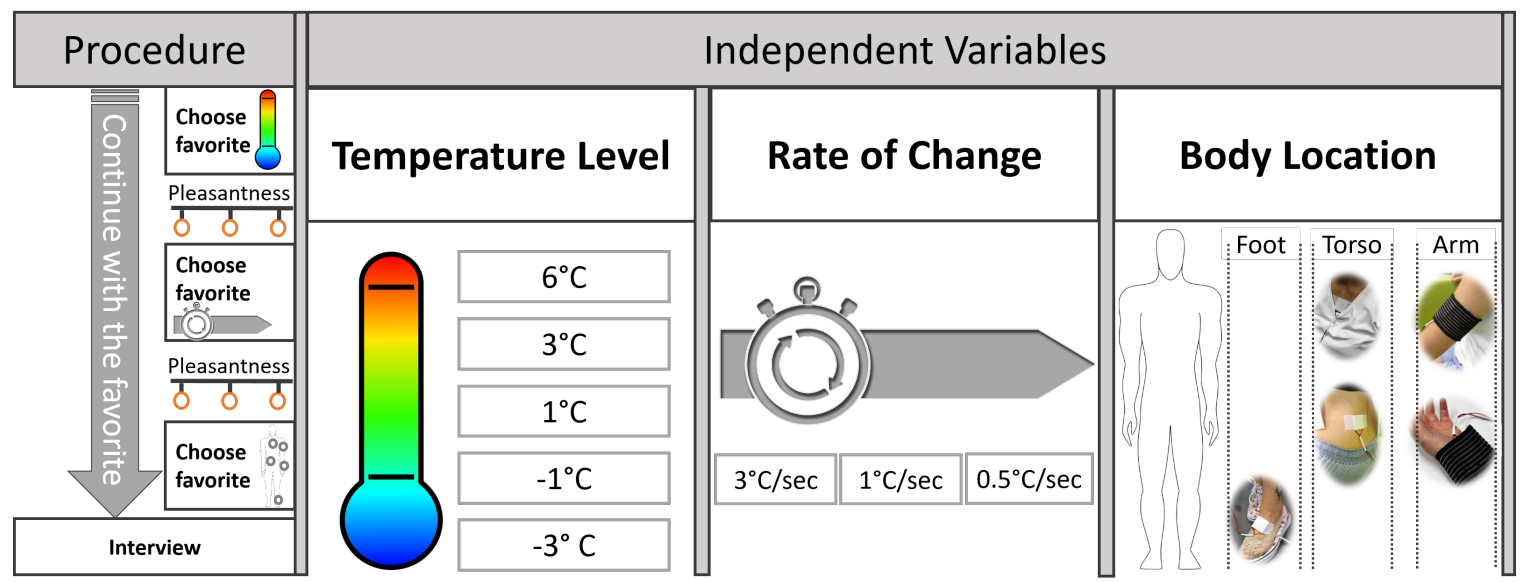

Figure 1: Illustration of the study procedure comprising the continuation with the favorite stimulus only and their stimuli.

\subsection{Apparatus}

Our apparatus consisted of the temperature stimuli delivering hardware prototype, the set of quantitative and qualitative questions.

Hardware Prototype. We built a hardware prototype (see Figure 2) which provides thermal feedback by conducting heat through a Peliter element (TEC1-12706) of $40 \mathrm{~mm} \times 40 \mathrm{~mm} \times 3.9 \mathrm{~mm}$ (cf. Figure 24). The Peliter element was connected to a L298N motor controller (cf. Figure 22) to control the voltage and current direction, hence control the rate of temperature change and values. The motor controller was powered by a DC power supply at constant voltage of $15 \mathrm{~V}$. The motor was controlled by an Arduino UNO. The Arduino UNO was connected to a laptop, which ran a controlling software program written in $\mathrm{C}++$. This program adjusted the temperature value of the Peltier element.

Questionnaires. For assessing pleasantness, we asked the participants to rate "How pleasant would that temperature level be" on a five-point Likert scale ranging from "Very unpleasant" (=1) to "Very

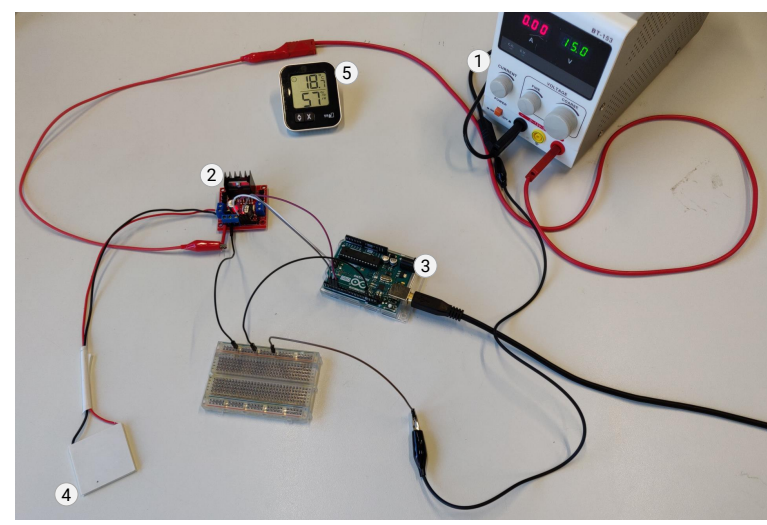

Figure 2: Hardware prototype providing thermal feedback consisting of DC power supply (1), motor driver (2), Arduino (3), peltier stimulator (4), thermometer, and hygrometer (5). pleasant" (=5). This question was introduced by the sentence "If you imagined receiving the thermal feedback during a stressful situation"; We used this scale for both, the temperature level and the rate of change evaluation. For comparing the discomfort and interference with the ability to work among the five body locations, we used two out of three items from the Cornell Hand Discomfort Questionnaire [6]. The first question was "If you experienced ache, pain, discomfort, how uncomfortable was this?" being rated on a three-point Likert scale (1="Slightly uncomfortable", 2="Moderately uncomfortable", $3=$ "Very uncomfortable"). The second question also contained a three-point Likert scale but provided the answers "Not at all" $(=0)$, "Slightly interfered" (=1), and "Substantially interfered" (=2).

Qualitative Questions. Additional to the questions asked to compare the feedback parameters, we conducted a short semi-structured interview after the study procedure. For this, we wanted to know if the participants would perceive thermal feedback as helpful in stressful situations and what they generally thought of thermal feedback. Further, we were interested in why they had chosen the preferred temperature level, rate of change, and body location and if could image other body locations to be useful for this purpose. Lastly, we asked them what they considered as advantages or disadvantages of thermal feedback for stress notification, particularly when compared to other feedback methods.

\subsection{Study Design and Variables}

Evaluating different temperature levels, rates of change, and body locations, these were our independent variables. We compared five temperature levels $\left(-3^{\circ} \mathrm{C},-1^{\circ} \mathrm{C}, 1^{\circ} \mathrm{C}, 3^{\circ} \mathrm{C}, 6^{\circ} \mathrm{C}\right)$ and three rates of change $\left( \pm 1^{\circ} \mathrm{C} / \mathrm{s}, \pm 3^{\circ} \mathrm{C} / \mathrm{s}, \pm 0.5^{\circ} \mathrm{C} / \mathrm{s}\right)$, we tested five body locations (wrist, upper arm, lower back, upper chest, foot arch) all presented to the participants in randomized order (cf. Figure 1). We followed a within-subject design assessing the pleasantness for the temperature and rate of change, while for the body locations we measured perceived discomfort and interference. Hence, these were our dependent variables specifically for the evaluating the preference of thermal feedback. 


\begin{tabular}{|c|c|c|c|c|}
\hline & $\begin{array}{c}\text { Temperature } \\
\text { Levels }\end{array}$ & \multicolumn{2}{|c|}{$\begin{array}{c}\text { Body } \\
\text { Locations }\end{array}$} & \\
\hline $6^{\circ} \mathrm{C}$ & $\begin{array}{l}\text { Pleasantness } \\
2.52(1.16)\end{array}$ & $\begin{array}{l}\text { Discomfort } \\
0.33(0.58)\end{array}$ & $\begin{array}{l}\text { Interference } \\
0.45(0.6)\end{array}$ & Wrist \\
\hline $3^{\circ} \mathrm{C}$ & $2.48(1.29)$ & $0.48(0.75)$ & $0.62(0.59)$ & Arm \\
\hline $1^{\circ} \mathrm{C}$ & $3.43(0.98)$ & $0.33(0.77)$ & $0.33(0.69)$ & Chest \\
\hline$-1^{\circ} \mathrm{C}$ & $3.95(0.86)$ & $0.29(0.72)$ & $0,48(0.75)$ & Back \\
\hline$-3^{\circ} \mathrm{C}$ & $3.48(1.33)$ & $0.37(0.6)$ & $0.47(0.6)$ & Foot \\
\hline
\end{tabular}

Table 1: The results (means and standard deviations in parantheses) for the temperature level pleasantness rating and the evaluation of body locations according to discomfort and interference with the ability to work. Before taking the preferred one for comparing it among all five body locations, each participant received each temperature level.

\subsection{Participants and Procedure}

We recruited 21 participants (9 females) with a mean age of 23.4 years $(S D=3.5)$. They were acquired via university mailing lists and personal contacts. After the participants were explained the study purpose and given the consent form reassuring that they agreed to have their answers audio recorded, we asked them to imagine a stressful situation which elicits stress specifically for themselves. We then attached the Peltier element fixed with a bandage having a velcro strap while collecting demographic data. Subsequently we started providing five different temperature levels asking to rate their pleasantness for each right after being presented. For assessing the favorite rate of change, we took the temperature level the participant previously had rated best and continued evaluating three rates of change. We then asked the participants to attach the Peltier element to the five different body locations successively intermitted by the specific ratings of discomfort and interference for each body location. Hereby, again we presented the temperature level and rate of change they had rated best before. Before we thanked them, we conducted a short semi-structured interview. The order of the different stimulus was counterbalanced using a Latin square. The study took approximately 30 minutes for each participant and they were compensated with sweets.

\section{RESULTS}

Next, we present our evaluation results leading to find the preferred thermal feedback for notifying users about stress.

Quantitative. For the temperature level we inferred that cold stimuli were perceived more pleasant $(M=3.71, S D=0.98)$ than the warm temperature levels $(M=2.75, S D=0.92)$. While the most pleasant stimuli, $-1^{\circ} \mathrm{C}$ was rated highest $(M=3.95, S D=0.86)$, the least pleasant was also the warmest, $3^{\circ} \mathrm{C}(M=2.48, S D=1.29)$. The exact ratings for the other stimuli can be obtained from Table 1 . Comparing the three rates of change, we found that the lower rates were perceived better. Accordingly, $\pm 0.5^{\circ} \mathrm{C} / \mathrm{s}$ was rated highest $(M=3.86, S D=1.11)$ followed by $\pm 1^{\circ} \mathrm{C} / \mathrm{s}(M=3.57, S D=0.93)$ and $\pm 3^{\circ} \mathrm{C} / \mathrm{s}(M=3.14, S D=1.11)$. Since we took the preferred stimuli individually for each participant and continued the study procedure with it, we found that five participants chose the warm temperature and the rest preferred to succeed with the cold stimuli, particularly nine participants decided for a temperature level of $-1^{\circ} \mathrm{C}$. Regarding the preferred body location for presenting thermal feedback, we obtained that our participants preferred the lower back and thus, found it least discomfortable $(M=0.29, S D=0.72)$. The other body locations were rated similarly (cf. Table 1); Five participants did not feel any discomfort when having presented the thermal feedback at any of these locations. Additionally, we were interested if the wearers felt any interference with their ability to work when receiving the thermal feedback. While mostly no or a very light inference was reported for the locations, we observed that the upper arm was rated highest $(M=0.62, S D=0.59)$ and five participants particularly commented on this location when being asked about a possible interference. The exact ratings are depicted in Table 1.

Inferential Statistical Analysis. We tested the effect of temperature levels on the perceived pleasantness indicated by the Likert scale, using Friedman test we found a statistically significant difference $\left(X^{2}(80)=30.88, p<0.0001\right)$.When comparing the cold and warm stimuli of the same temperature level, based on Wilcoxon signedrank test there was no significant difference $(Z=27.5, p=0.56)$. Whereas another Wilcoxon signed-rank test between temperature levels $-3^{\circ} \mathrm{C}$ and $3^{\circ} \mathrm{C}$ showed a significant difference $(Z=25.5, p=$ 0.026 ) between the perceived pleasantness of the thermal stimuli. Further, we tested the effect of the rate of change on the pleasantness ratings. The Friedman test suggested a statistically significant difference $\left(X^{2}(40)=7.12, p=0.03\right)$. A post-hoc analysis applying a Wilcoxon signed-rank test indicated that there is no statistically significant difference in the pleasantness rating between the rates of change of $\pm 0.5^{\circ} \mathrm{C} / \mathrm{s}$ and $\pm 1^{\circ} \mathrm{C} / \mathrm{s}$, nor between $\pm 1^{\circ} \mathrm{C} / \mathrm{s}$ and $\pm 3^{\circ} \mathrm{C} / \mathrm{s}$. However, a Wilcoxon signed-rank test between the pleasantness rating for the rates of change of $\pm 0.5^{\circ} \mathrm{C} / \mathrm{s}$ and $\pm 3^{\circ} \mathrm{C} / \mathrm{s}$ showed a significant difference $(Z=17.5, p=0.01)$. A Friedman test to compare the discomfort rating on the Likert scale between the tested body locations, did not indicate a statistically significant difference $(p=0.5)$. A Friedman test to compare the interference rating on the Likert scale between the different body locations, did not indicate a statistically significant difference $(p=0.82)$.

Qualitative Results. On the general idea of being notified by thermal feedback on one's stress level, 18 out of 21 commented positively. Only two participants stated that they did not see the benefit from thermal feedback and one participant even criticized it to be unpleasant in stressful situations. When being asked particularly on their considerations about thermal feedback signaling stress, three interviewees said they would appreciate such feedback in case it is not distracting in the very moment. Further, we aimed to understand why our participants chose the temperature level, rate of change, and body locations as they did. Accordingly, the 16 participants who preferred the cold stimuli explained that they usually feel hot when being stressed and thus, the counter stimulus might help them to cool down and respectively to cope better with the situation. Another interesting explanation was given by one interviewee stating that he perceived the cold stimulus as unusual in comparison to the heated devices he wears on his body, such as the smartphone and smartwatch. For choosing the rate of change, three participants preferred a low rate to avoid being distracted by 
a faster change. In contrast, five participants worried that having a less intense temperature level or a low rate of change, they would not notice the thermal feedback under stress and consequently be irritated because they expected it then. Regarding the body locations, one third would appreciate to receive thermal feedback at the wrist. Four of them wore a smartwatch and loved the idea to integrate thermal feedback in existing wrist-worn wearables. The second favorite location according to the qualitative data was the upper arm, as chosen by three interviewees. The upper chest and likewise the foot were preferred by one participant each. Almost half of our sample did not state any preference on the body location, while one participant explicitly disliked the lower back. As an advantage of thermal feedback, eight participants stated that such feedback preserves privacy, which becomes even more important when signaling stress. Another two mentioned the advantage that other feedback channels, namely audio or vibration are already occupied by other devices and therefore thermal feedback has a unique value making it easy to identify its source and meaning. One additional remark was referring to the quality of unobtrusiveness of thermal feedback; One participant said that it felt like more "direct" coming from his own body and not being induced externally.

\section{DISCUSSION AND RESEARCH AGENDA}

Before we will conclude from our study, we discuss our findings briefly and present our research agenda to be realized in the future.

\subsection{Discussion}

Informed by previous work, thermal feedback perception is highly subjective and includes various variables, such as the temperature level, rate of change as well as the body location where the feedback is presented. Hence, we conducted a preliminary study to identify users' preferences for these main influencing factors. As can be obtained from the results, the perception of thermal is highly subjective and prone to personal preferences. For instance, one participant rated all temperature levels as unpleasant and another did vice versa. This highlights that thermal feedback is a personalized feedback channel that does not follow "one size fits all" configuration, which is in line with previous work. Further, prior work shows that thermal cues are conveying emotions [14] and are rated in dependency to context factors [4]. Our findings validate the influence of the variables of thermal feedback on both the comfort and interference. Interestingly, the qualitative results are not necessarily reflecting how people rated the different stimuli in the questionnaires. Most striking is the preference on the body location. Although the mean ratings are very similar for each of them and did not show any significant difference, in the interviews, participants stated that the wrist or upper arm would be a suitable location despite the upper arm was rated to have the most discomfort $(M=0.48)$ and the highest interference with the ability to work $(M=0.62)$.

\subsection{Research Agenda}

Based on our results, we built a mobile prototype being able to deliver distinct temperature levels with different rates of change. Since we deduced from the participants' feedback that the lower back was rated as the optimal location, we designed the portable prototype such, that two Peltier elements could be attached to each side of the lower back (right and left bottom). The Arduino and battery packs as power supply are hidden in a belt bag. With the following research agenda we plan to expand the research on thermal feedback for notifying about stress:

- We plan to refine the portable prototype in terms of battery consumption, weight, etc..

- With the finalized prototype we plan to conduct a field study involving a realistic stress situation where users receive insitu thermal feedback on their stress level.

- In the field study we plan to assess three different aspects, namely the user acceptance, user experience, and practicability of our prototype and concept.

- Finally we aim to conclude from the field's study result how thermal feedback on the stress level is perceived by users and what it needs to be put into practice

\section{CONCLUSION}

By this work we investigated the general suitability and preferences of thermal feedback for notifying users about their stress level. Therefore, we evaluated three different measures, the temperature level, rate of change, and body location. For each of them we compared distinct stimuli in a user study involving 21 participants. From both, the quantitative and qualitative results, we observed that users appreciate the idea of thermal feedback as a notifier about stress given its advantages of privacy preservation and unobtrusiveness. Further, they were interested in incorporating it in existing technologies, such as smartwatches. We further found that cold stimuli, in particular $-1^{\circ} \mathrm{C}$ with a change rate of $\pm 0.5^{\circ} \mathrm{C} / \mathrm{s}$ are preferred for presenting feedback. Regarding the best body location, we got ambiguous feedback. While participants liked the possibility of using familiar locations, namely the wrist, they feared it could interfere with their ability to work on the other hand. The upper back seemed to avoid this issue and support the privacy quality being mentioned as an considerable aspect. We conclude this paper with our research agenda being inspired from the presented results.

\section{ACKNOWLEDGMENTS}

This project was supported by the German Research Foundation (DFG) within the Transregio SFB 161 C02 project number 251654672 and was partly conducted within the AMPLIFY project funded from the European Research Council (grant no. 683008).

\section{REFERENCES}

[1] Y. Abdelrahman, E. Velloso, T. Dingler, A. Schmidt, and F. Vetere. 2017. Cognitive heat: exploring the usage of thermal imaging to unobtrusively estimate cognitive load. Proc. of the ACM on Interactive, Mobile, Wearable and Ubiquitous Technologies 1, 3 (2017), 33

[2] Yasmeen Abdrabou, Khaled Kassem, Jailan Salah, Reem El-Gendy, Mahesty Morsy, Yomna Abdelrahman, and Slim Abdennadher. 2018. Exploring the Usage of EEG and Pupil Diameter to Detect Elicited Valence. In International Conference on Intelligent Human Systems Integration. Springer, 287-293.

[3] M. ElKomy, Y. Abdelrahman, M. Funk, T. Dingler, A. Schmidt, and S. Abdennadher. 2017. ABBAS: an adaptive bio-sensors based assistive system. In Proc. of the 2017 CHI Conf. Extended Abstracts on Human Factors in Computing Systems. ACM, 2543-2550.

[4] D. Gooch. 2009. An Investigation into Communicating Social Presence With Thermal Devices. Ph.D. Dissertation. MSc Dissertation.

[5] K. Hänsel, R. Poguntke, H. Haddadi, A. Alomainy, and A. Schmidt. 2018. What to Put on the User: Sensing Technologies for Studies and Physiology Aware Systems. In Proc. of the 2018 CHI Conf. on Human Factors in Computing Systems (CHI '18). 
ACM, New York, NY, USA, Article 145, 14 pages. http://doi.acm.org/10.1145/ 3173574.3173719

[6] A. Hedge, S. Morimoto, and D. Mccrobie. 1999. Effects of keyboard tray geometry on upper body posture and comfort. Ergonomics 42, 10 (1999), 1333-1349. https: //doi.org/10.1080/001401399184983 PMID: 10582503.

[7] Khaled Kassem, Jailan Salah, Yasmeen Abdrabou, Mahesty Morsy, Reem ElGendy, Yomna Abdelrahman, and Slim Abdennadher. 2017. DiVA: Exploring the Usage of Pupil L’łUL'ńDiL’ł/UL'ńAmeter to Elicit L'łUL'ńVL'ł/UL'ńAlence and LłłUL'ńaL'ł/UL'ńRousal. In Proceedings of the 16th International Conference on Mobile and Ubiquitous Multimedia (MUM '17). ACM, New York, NY, USA, 273-278. https://doi.org/10.1145/3152832.3152836

[8] R. Kettner, P. Bader, T. Kosch, S. Schneegass, and A. Schmidt. 2017. Towards Pressure-based Feedback for Non-stressful Tactile Notifications. In Proc. of the 19th Intern. Conf. on Human-Computer Interaction with Mobile Devices and Services (MobileHCI '17). ACM, New York, NY, USA, Article 89, 8 pages. https://doi.org/ $10.1145 / 3098279.3122132$

[9] E. Labbé, N. Schmidt, J. Babin, and M. Pharr. 2007. Coping with stress: the effectiveness of different types of music. Applied psychophysiology and biofeedback 32, 3-4 (2007), 163-168.
[10] W. Lee and Y. Lim. 2010. Thermo-message: exploring the potential of heat as a modality of peripheral expression. In CHI'10 Extended Abstracts on Human Factors in Computing Systems. ACM, 4231-4236.

[11] E.R. Nadel, J.W. Mitchell, and J.A.J. Stolwijk. 1973. Differential thermal sensitivity in the human skin. Pflügers Archiv 340, 1 (1973), 71-76.

[12] P. Paredes, D. Sun, and J. Canny. 2013. Sensor-less Sensing for Affective Computing and Stress Management Technology. In Proc. of the 7th Intern. Conf. on Pervasive Computing Technologies for Healthcare (PervasiveHealth '13). ICST (Institute for Computer Sciences, Social-Informatics and Telecommunications Engineering), ICST, Brussels, Belgium, Belgium, 459-463. http://dx.doi.org/10.4108/icst. pervasivehealth.2013.252380

[13] Jailan Salah, Yomna Abdelrahman, Yasmeen Abdrabou, Khaled Kassem, and Slim Abdennadher. 2018. Exploring the Usage of Commercial Bio-Sensors for Multitasking Detection. In Proceedings of the 17th International Conference on Mobile and Ubiquitous Multimedia (MUM 2018). ACM, New York, NY, USA, 265277. https://doi.org/10.1145/3282894.3282900

[14] G. Wilson, M. Halvey, S.A. Brewster, and S.A. Hughes. 2011. Some like it hot: thermal feedback for mobile devices. In Proc. of the SIGCHI Conf. on Human Factors in Computing Systems. ACM, 2555-2564. 